\title{
Qualitative and Quantitative Histomorphologic Assessment of Fathead Minnow Pimephales promelas Gonads as an Endpoint for Evaluating Endocrine-Active Compounds: A Pilot Methodology Study
}

\author{
JefFrey C. Wolf, ${ }^{1}$ Daniel R. Dietrich, ${ }^{2}$ Urs Friederich, ${ }^{3}$ John CAUnter, ${ }^{4}$ AND ANDrew R. Brown ${ }^{4}$ \\ ${ }^{1}$ EPL, Inc., 22866 Shaw Road, Sterling, VA 20166, USA \\ ${ }^{2}$ Environmental Toxicology, University of Konstanz, Germany \\ ${ }^{3}$ Dow Europe Gmbh, Switzerland, and \\ ${ }^{4}$ Brixham Env. Laboratory, AstraZeneca UK Ltd., Brixham, U.K.
}

\begin{abstract}
Although histopathology is routinely employed as a tool for the detection and assessment of xenobiotic-mediated effects in mammals, it is less frequently applied to fish. In part, this is due to a lack of method standardization regarding study design, tissue preservation, tissue sectioning, histopathological evaluation, reporting, and statistical analysis. The objectives of the present study were: (1) to test and refine a method for the microsurgical excision of fathead minnow (FHM) Pimephales promelas gonads for the purpose of histopathologic examination; (2) to determine the optimal combination of fixation and embedding procedures for the histopathologic and morphometric analysis of FHM gonads following exposure to a known estrogenic compound, $17 \beta$-estradiol (E2); and (3) to provide a method for the categorization and quantification of cell types in FHM gonads by manually counting cells in digitized images using image analysis software. The light microscopic evaluation of individual gametogenic cells was greatly facilitated by specimen preparation techniques that included the excision of gonads via microdissection and by optimized fixation and embedding procedures.
\end{abstract}

Keywords. Endocrine-active; reproduction; fathead minnow; Pimephales promelas; testis; ova; morphometry; histopathology.

\section{INTRODUCTION}

Fish are suitable models for evaluating endocrine-active compounds in the water column. Fish are exposed to waterborne chemicals simultaneously via the respiratory, digestive, and dermal routes, and the short reproductive cycle of small fish species ensures that chemical effects on reproduction are swiftly apparent. Perhaps most importantly, it has been demonstrated that various fishes undergo physiological and morphological changes in response to putative endocrine disrupters (Christiansen et al., 1998; Miles-Richardson et al., 1999a; Ankley et al. 2001; Van den Belt et al., 2002; van der Ven and Wester, 2002; Kinnberg and Toft, 2003; van der Ven et al., 2003).

A variety of endpoints have been utilized to investigate the effects of hormones and hormone mimics on the fish reproductive system. Examples include changes in plasma levels of vitellogenin and sex steroids such as $17 \beta$-estradiol and 11-ketotestosterone, alterations in the external morphology of sexually dimorphic fishes, variations in gonado-somatic indices (ratios of gonad weight to body weight), and reproductive fecundity. Although histopathology is routinely employed as a tool for the detection and assessment of xenobiotic-mediated effects in mammals, it is less frequently

\footnotetext{
${ }^{1}$ Abbreviations: FHM, fathead minnow; E2, $17 \beta$-estradiol; NBF, neutral buffered formalin; GIP, grid intersection points; VC, vacuolated cell; and $\mathrm{ABC}$, apoptotic body cell.

Address correspondence to: Dr. Jeffrey C. Wolf, Experimental Pathology Laboratories, Inc., 22866 Shaw Road, Sterling, Virginia 20166, USA; e-mail: jwolfepl@aol.com
}

applied to fish. In part, this is due to a lack of method standardization regarding study design, tissue preservation, tissue sectioning, histopathological evaluation, reporting, and statistical analysis, primarily related to a lack of regulatory requirements mandating the examination of fish tissues. Consequently, reliable and readily comparable results have not been achieved consistently.

The histopathological assessment of fish reproductive organs can be divided into 2 separate components: the evaluation of gonads for abnormal findings (examples of which include such varied observations as necrosis, Sertoli cell hypertrophy/hyperplasia, and the occurrence of testicular oocytes), and gonad staging. Gonad staging involves the assessment of germinal cell type proportions in order to identify potential effects of exogenous or endogenous chemicals on gametogenesis. Gonad staging can be performed quantitatively or semiquantitatively; the latter is more commonly reported, and different semiquantitative staging schemes have been described (Miles-Richardson et al., 1999a; Nichols et al., 2001; Jensen et al., 2001; Ankley et al., 2002; Van den Belt et al., 2002; U.S. EPA, 2002).

Because semiquantitative systems primarily assess the visual density of gametogenic precursors compared to mature gametocytes, a shortcoming inherent to such systems is a limitation in the types of data that may be obtained. As an example, the loss or overabundance of a specific gametogenic stage may not be apparent using some semiquantitative systems. Such shortcomings may be mitigated by using a quantitative staging approach in which gonadal cell types are individually identified and counted. Furthermore, a distinct advantage of 
quantitative histomorphometry is the ability to apply statistical methods to the data in order to discern subtle treatmentrelated effects that might otherwise be difficult to appreciate. There are several reports in which quantitative schemes were used for the staging of fish gonads (Smith, 1978; Department of the Interior, 2000; Sohoni et al., 2001; van der Ven et al., 2003). In contrast to semiquantitative staging, a key requirement for quantitative staging is that each counted cell must be assigned to one of several well-defined categories according to predetermined histomorphologic criteria. Consequently, in order to adequately visualize and differentiate the different gonadal cell types, the tissue collection procedures and the preparation of the histologic sections need to be optimized-enhanced cellular detail is especially important for distinguishing the relatively small germinal cells of the fish testis. Therefore, the objectives of the present study were: (1) to test and refine a method for the microdissection of fathead minnow (FHM $)^{1}$ gonads for the purpose of histopathologic examination; (2) to determine the optimal combination of fixation and embedding procedures for the histopathologic and morphometric analysis of fathead minnow gonads following exposure to a known estrogenic compound, $17 \beta$-estradiol (E2); and (3) to provide a method for the categorization and quantification of cell types in FHM gonads by manually counting cells in digitized images using image analysis software.

\section{MATERIALS AND METHODS}

\section{Study Design and Exposure of Fish to the Test Substance}

The study design is summarized in Table 1 . Briefly, 9 male and 9 female fathead minnows, Pimephales promelas, were continuously exposed to $10 \mathrm{nM} 17 \beta$-estradiol $(2780 \mathrm{ng} / \mathrm{l})$ during the 10-day study. Because documentation of $17 \beta$ estradiol effects per se was not a primary aim of this pilot project, negative (diluent water) control fish were not incorporated into the design of the present study, and the overall number of fish was kept to a minimum. All FHM were approximately 160 days posthatch at the start of the study, and the mean length/weight measurements at terminal sacrifice were $64 \mathrm{~mm} / 5.73 \mathrm{~g}$ for males, and $47 \mathrm{~mm} / 1.99 \mathrm{~g}$ for females. The test substance, $17 \beta$-estradiol, was obtained from Sigma Aldrich Chemical Company (product number E8875, lot number 070K1206). The certificate of analysis accompanying the test substance confirmed a purity of $100 \%$. A single stock solution of $5.56 \mathrm{mg} \mathrm{L}^{-1}$ was prepared in ethanol $(90 \% \mathrm{v} / \mathrm{v})$ and was stirred continuously during the test. This stock solution was delivered to a mixing chamber at a nominal flow rate of $0.125 \mathrm{ml} \mathrm{min}^{-1}$, along with dilution water, at a nominal flow rate of $250 \mathrm{ml} \mathrm{min}{ }^{-1}$, giving a nominal

TABLE 1.-Distribution of fish numbers, fixation methods, and embedding media.

\begin{tabular}{llccc}
\hline \hline & & $\begin{array}{c}17 \beta \text {-estradiol } \\
\text { exposure } \\
\text { Method of fixation }\end{array}$ & Embedding medium & \multicolumn{2}{c}{ Numbers of fish } \\
\cline { 4 - 5 } & concentration & Males & Females \\
\hline Formalin $^{a}$ & Paraffin wax & $2,780 \mathrm{ng} \mathrm{L}^{-1}$ & 3 & 3 \\
Bouin's solution $^{b}$ & Paraffin wax & $2,780 \mathrm{ng} \mathrm{L}^{-1}$ & 3 & 3 \\
Glutaraldehyde $^{b}$ & Glycol methacrylate & $2,780 \mathrm{ng} \mathrm{L}^{-1}$ & 3 & 3 \\
\hline
\end{tabular}

${ }^{a} 10 \%$ neutral buffered formalin.

${ }^{b} 5 \%$ glutaraldehyde, unbuffered. dilution, immediately before delivery into the test vessel, of 2000:1. This dilution gave the required nominal test concentration of $2780 \mathrm{ng} \mathrm{L}^{-1}$, along with an ethanol $(90 \% \mathrm{v} / \mathrm{v})$ concentration of $500 \mu \mathrm{L} \mathrm{L}^{-1}$. The dilution water was dechlorinated tap water supplied from a $100 \mathrm{~m}^{3}$ reservoir with an average retention time of 24 hours. It was passed through activated carbon, coarsely filtered to remove particulate material, dechlorinated with sodium thiosulphate, and salts added, as required, to maintain minimum hardness levels. The treated water was held in a secondary reservoir with a capacity of $36 \mathrm{~m}^{3}$ (Christiansen, Korsgaard, and Jespersen (1998) and an average retention time of 8 hours. The water was passed through an ultraviolet sterilizer to a second set of filters (25 and $10 \mu \mathrm{m}$ mesh size) and then to a third storage tank with a capacity of $13.5 \mathrm{~m}^{3}$ (Christiansen, Korsgaard, and Jespersen (1998). The treated water was delivered via a ring circuit to a temperature-controlled header tank in the test laboratory set to a nominal temperature of $25 \pm 1^{\circ} \mathrm{C}$ and finally passed through a $5 \mu \mathrm{m}$ filter before use. The volume of test solution in which fish were exposed was $57 \mathrm{~L}$, giving a maximum loading of $6.0 \mathrm{~g}$ of fish per liter of solution, and the total flow was equivalent to a minimum of $1 \mathrm{~L}$ per $\mathrm{g}$ of fish per day. Analysis of $17 \beta$-estradiol concentrations was performed using an estradiol enzyme immunoassay kit (Cat. No. 582251, Cayman Chemical Company, Ann Arbor, Michigan, USA). Calculated from the day 0 to day 10 results, the mean of the measured $17 \beta$-estradiol exposure concentration was $90 \%$ of the nominal concentration (range, $79-115 \%$ of nominal). Exposures were conducted at a temperature of $25^{\circ} \mathrm{C} \pm 1{ }^{\circ} \mathrm{C}$, and the photoperiod was 16 hours of light followed by 8 hours of darkness, with a 20-minute dawn-and-dusk transition period, respectively. The exposure tanks were aerated to maintain sufficient oxygen levels. Test conditions monitored during the study included dissolved oxygen $\left(\mathrm{mg} \mathrm{L}^{-1}\right), \mathrm{pH}$, temperature $\left({ }^{\circ} \mathrm{C}\right)$, dilution water flow $\left(\mathrm{ml} \mathrm{L}^{-1}\right)$, toxicant flow $(\mathrm{ml}$ $\mathrm{L}^{-1}$ ), and dilution ratio. The dilution water (dechlorinated and filtered to $5 \mu \mathrm{m}$ ) was also analyzed for hardness and conductivity once weekly.

\section{Euthanasia and Necropsy}

At the end of the test period, each of the 18 fish was individually captured, euthanized by an overdose of tricaine methanesulfonate (MS222, 3-aminobenzoic acid ethyl ester methanesulfonate $500 \mathrm{mg} / \mathrm{l}$; buffered to $\mathrm{pH} 7-8$ using $1 \mathrm{M}$ $\mathrm{NaOH}$ ), followed by cervical severance, and then immediately necropsied. Individual fish were selected for euthanasia and necropsy at random, alternating male with female fish.

Each fish was necropsied by placing it in right lateral recumbency on the stage of a dissecting microscope. Fine dissecting instruments were used to remove the left body wall and to excise the gonads by severing the spermatic ducts or oviducts and mesenteric attachments. All of the gonads were dissected in a caudal to cranial direction. Macroscopic external or internal lesions were noted for individual fish. The left and right gonads from each fish were placed into separate compartments of a plastic tissue cassette and assigned together to 1 of 3 fixation solutions, in rotating fashion, according to the order in which the fish were necropsied. The 3 fixation solutions used in this study were: $10 \%$ neutral buffered formalin (NBF); Bouin's solution (71\% saturated picric acid, 
24\% formaldehyde, 5\% glacial acetic acid; Sigma-Aldrich, St. Louis, Missouri, USA); and 5\% glutaraldehyde in deionized water (Table 1). At 24 hours following tissue collection, cassettes placed in Bouin's solution were rinsed in 70\% ethanol and then transferred to containers of $70 \%$ ethanol.

\section{Tissue Processing, Embedding, and Microtoming}

Left and right gonads from each of the 18 fish were processed routinely according to standard histologic methods, and then embedded as follows: gonads fixed in formalin or Bouin's solution were embedded in paraffin (Paraplast, Tyco Healthcare Group, Mannfield, Massachusetts, USA); whereas, gonads fixed in glutaraldehyde were embedded in glycol methacrylate (GMA, Polysciences, Warrington, Pennsylvania, USA) (Table 1). The gonads in block form were trimmed to their largest longitudinal sectional area, and then sections (each section approximately $3-5 \mu \mathrm{m}$ thickness for paraffin-embedded tissues or 1-2 $\mu$ m thickness for GMA) from each of the 18 fish were microtomed from each gonad, placed on individual glass slides, and stained with hematoxylin and eosin (H\&E).

\section{Qualitative Histomorphologic Assessment}

The suitability of the various fixation and embedding combinations for the identification of individual gonadal cell types was evaluated subjectively via light microscopy. In addition, all gonad sections were examined for morphologic abnormalities and potential treatment-related changes. The severity of any inflammatory, degenerative, or proliferative changes was graded as minimal, mild, moderate, moderately severe, or severe. Macroscopic observations were correlated with microscopic findings whenever possible.

\section{Quantitative Histomorphologic Assessment}

This assessment involved the identification of gonad cell types and the counting (manual tagging) of individual gonad cells in digitized photomicrographs. Equipment used for the acquisition of images included an Olympus BX51 microscope, a SPOT Insight Color digital camera (Diagnostic Instruments, Inc., Sterling Heights, Michigan, USA) plus version 3.2 of the accompanying SPOT software, and an IBMcompatible Dell Dimension personal computer running the Microsoft Windows 2000 Professional operating system.

Optimal results for the testes were obtained from sections that were preserved in 5\% glutaraldehyde and embedded in GMA (see Results section); therefore, manual tagging of testes cell types was restricted to digital photomicrographs of these sections. Four digital images $(0.22 \mathrm{~mm} \times 0.29 \mathrm{~mm}$ subject area) were obtained from each of the left and right testis sections (i.e., 8 images total per fish) using the $40 \times$ microscope objective. To ensure the acquisition of nonoverlapping areas, an ultrafine permanent marker was used to create a grid (each grid square was $2 \times 2 \mathrm{~mm}$ ) on the underside of each glass slide (the overall size of the grid was created large enough to fully encompass each testis section). Areas for photography were selected from the centers of grid squares that were chosen arbitrarily. For each digital image, ImageProPlus (IPP-Media Cybernetics, Silver Spring, Maryland, USA, version 4.1) was used by a pathologist to manually tag individual testis cells as to cell type. Tagging was performed by a single pathologist to avoid inconsistencies. The contrast of each image was globally enhanced using IPP's "Best Fit" equalization algorithm. A virtual grid consisting of $400(20 \times$ 20 ) individual intersection points was applied to each image as an overlay. Grid intersection points (GIP) were separated from one another by $0.015 \mathrm{~mm}$ horizontally and $0.011 \mathrm{~mm}$ vertically.

Each GIP was tagged with 1 of 6 different-colored dots to represent one of the following cell categories (for criteria see the Results section): spermatozoon, spermatid, spermatocyte, spermatogonium, vacuolated cell (VC), or apoptotic body cell (ABC). All GIP were tagged, with the following exceptions: GIP that covered empty space in extracellular areas, GIP that covered interstitial structures (collagen, blood vessels), or GIP that covered nonspermatogenic cells or cells that could not be identified (e.g., due to tangential sectioning of the cells or tissue artifact). A minimum of 2,000 spermatogenic cells total were counted per male fish. A grid was used to count cells in the testis because it provided a nonbiased method for subsampling a tissue that contains a large number of very small cells. No attempt was made to extrapolate these counts to any type of volumetric (i.e., stereological) measurement.

Optimal results for the ovaries were obtained from sections that were preserved in Bouin's solution and embedded in paraffin (see Results section); therefore, the manual tagging of ovarian cell types was restricted to images of sections made with this procedural combination. Two digital images $(2.2 \mathrm{~mm} \times 2.9 \mathrm{~mm}$ subject area) were obtained from each of the left and right ovarian sections (i.e., 4 images total per fish) using the $4 \times$ microscope objective. To ensure the acquisition of nonoverlapping areas, an ultrafine permanent marker was used to draw a line on the underside of the glass slide that approximately bisected the ovary section perpendicular to its long axis. The 2 tissue areas for photography, obtained from each half of the ovary, were selected arbitrarily. For each digital image, IPP was used by a pathologist to manually tag individual ovarian cells as to cell type. The contrast of each image was globally enhanced using IPP's "Best Fit" equalization algorithm. Via the computer mouse, the pathologist then sequentially tagged each ovarian follicle with 1 of 6 different solid-colored squares that each represented 1 of the 6 following follicle cell types: perinucleolar, cortical alveolar, early vitellogenic, late vitellogenic, mature/spawning, and atretic. A minimum of 100 follicles total were counted per female fish. Counts per image of the different cell types were exported to a Microsoft Excel worksheet and percentages were calculated for each cell type per gonad (testis or ovary, left and right) and per fish. To avoid having to make geometric assumptions concerning the shapes of cells or organs, the results were maintained as proportions (e.g., number of perinucleolar oocytes per total oocytes counted), without any attempt to convert the counts to stereological or volumetric estimates (e.g., number of perinucleolar oocytes per unit volume of ovary). Because the distribution of oocytes in cyprinid fishes such as fathead minnows is essentially random, a single section through the gonads is considered to be representative. This is In contrast to the situation in atheriniform fishes (medaka, for example) that have peripheral to central and cranial to caudal gradients of cell development in their gonads; an alternative approach to assessing cell populations would be necessary to avoid a sampling bias for that species. 
TABLE 2.-Histopathologic correlates to macroscopic findings in the gonads of fathead minnows exposed to $17 \beta$-estradiol.

\begin{tabular}{lll}
\hline \hline & \multicolumn{1}{c}{ Macroscopic finding } & Histopathologic correlate \\
\hline Testis & Attenuation & No specific correlate \\
Ovary & Attenuation, gelatinous texture & Follicular atresia \\
\hline
\end{tabular}

\section{RESULTS \\ Necropsy and Macroscopic Findings}

As recognized in previous method development exercises, the use of a dissecting microscope was considered to be at least highly advantageous, if not essential, for the removal of FHM gonads to minimize trauma to these delicate tissues. Additionally, removal of the swimbladder prior to excision of the gonads facilitated the microdissection procedure, and it appeared that trauma was minimized by dissecting the gonads in a caudal-to-cranial direction, while applying very gentle traction to the spermatic ducts or oviducts.

Macroscopic findings (Table 2) included observations pertaining to relative size differences among the testes and ovaries, respectively. For the males, testis size differences could not be attributed to any specific histopathologic diagnoses. For the females, ovarian size was generally dependent upon the degree to which large follicles were present in each ovary. Several of the excised ovaries had a gelatinous appearance and texture which were attributed to varying levels of follicular atresia as determined subsequently by microscopic examination.

\section{Qualitative Histomorphologic Assessment}

The combination of glutaraldehyde fixation and GMA embedding was judged to be superior to the combinations of Bouin's or formaldehyde and paraffin wax for identifying individual gonad cells in the testis (Figure 1A, 1C, and 1E). Cytoplasmic and nuclear details of the spermatogonia, VC, and $\mathrm{ABC}$ were enhanced in the thin (approximately 1-2 $\mu \mathrm{m}$ ) GMA-embedded sections, and the cytoplasmic borders of individual cells could often be recognized. In contrast, fixation of the testes in Bouin's solution produced cells with less nuclear and far less cytoplasmic detail, and individual cell borders were usually indistinct due to the greater thickness of the paraffin sections (approximately 3-5 $\mu \mathrm{m}$ ). Overall, testis cell types could be adequately (but not optimally) distinguished in Bouin's/paraffin sections. Compared to the other fixatives, $10 \% \mathrm{NBF}$ was considered to be inadequate for the purpose of cell counting in the testis; consistent differentiation of individual cell types was virtually impossible due to the artifactual shrinkage and condensation of the spermatogenic precursor nuclei.

The combination of Bouin's solution fixation and paraffin embedding was considered to be the most satisfactory of the 3 preparation methods for the ovary (Figure 1B, 1D, and 1F). The cytological detail of follicles and supportive cells was actually superior in the glutaraldehyde/GMA sections when compared to Bouin's fixed sections; however, because ovarian follicles are vastly larger than spermatogenic cells, this increased level of detail did not facilitate the differential identification of follicle types. In addition, the Bouin's/paraffin sections provided comparatively better color contrast among the different follicle types and a more classic morphologic ap-
TABLE 3.-Qualitative histopathologic findings and associated severities in the gonads of fathead minnows exposed to $17 \beta$-estradiol.

\begin{tabular}{lll}
\hline \hline & \multicolumn{1}{c}{ Histopathologic finding } & \multicolumn{1}{c}{ Range of severity } \\
\hline Testis & Cellular debris in efferent ducts & Minimal-to-mild \\
& Germ cell syncytia & Minimal \\
& Granulomas & Minimal \\
& Mineralization & Minimal \\
& Mineralization in efferent ducts & Moderate \\
& Sperm necrosis & Minimal-to-mild \\
Ovary & Follicular atresia & Minimal-to-moderate \\
\hline
\end{tabular}

pearance (i.e., more similar to published photomicrographs) when compared to the glutaraldehyde/GMA sections. Formalin fixation was considered to be adequate for FHM ovarian follicle differentiation, although this technique was associated with minor artifactual distortions such as wrinkling of the vitelline envelope and dissociation of the follicles. Early evidence of oocyte atresia, such as minor perforations of the vitelline membrane, was more readily observed in the Bouin's-fixed sections when compared to sections fixed in formalin.

Histopathologic abnormalities (Table 3) that were diagnosed in the testes of male FHM were generally mild and included cellular debris in spermatic ducts, granulomas, mineralization within the germinal epithelium, mineralization in spermatic ducts, germ cell syncytia, and sperm necrosis. The sole histopathologic finding in female fish was follicular atresia. Atretic follicles characterized in the next section.

There appeared to be minimal, if any, damage to the testes or ovaries as a function of tissue collection. For example, although collapsed follicles were evident in all ovaries to varying degrees, such follicles almost invariably had changes within their supportive cells (e.g., intracytoplasmic yolk material, perifollicular macrophage aggregates) that suggested that their collapse was caused by antemortem degeneration (atresia) versus postmortem handling trauma.

\section{Quantitative Histomorphologic Assessment}

The goals of counting a minimum of 2,000 cells (covered by grid intersection points) for each male fish and 100 follicles for each female fish were easily achieved. The average number of tagged cells per male fish (both testes combined) was 2,712 , and the average for females (both ovaries combined) was 275 . The relative percentages of gonadal cell types per fish as obtained by manual tagging are presented graphically (Figures 2 and 3). The ability of the pathologist to magnify each image to the limits of image resolution was beneficial for the identification of individual cell types. This degree of magnification would not have been available if counts had been performed directly from glass slides using a $40 \times$ microscope objective.

As previously mentioned, manual tagging of images acquired from the testis (Figure 4) utilized glutaraldehyde/ GMA sections. Spermatozoa were readily identified as small (approximately $2 \mu \mathrm{m}$ diameter), dense, discrete, approximately circular, essentially acytoplasmic, deep basophilic cells that were present in large numbers within the lumina of spermatogenic lobules. Compared to spermatozoa, Spermatids were slightly larger (approximately 2-3 $\mu \mathrm{m}$ diameter) than spermatozoa, and had narrow rims of eosinophilic 


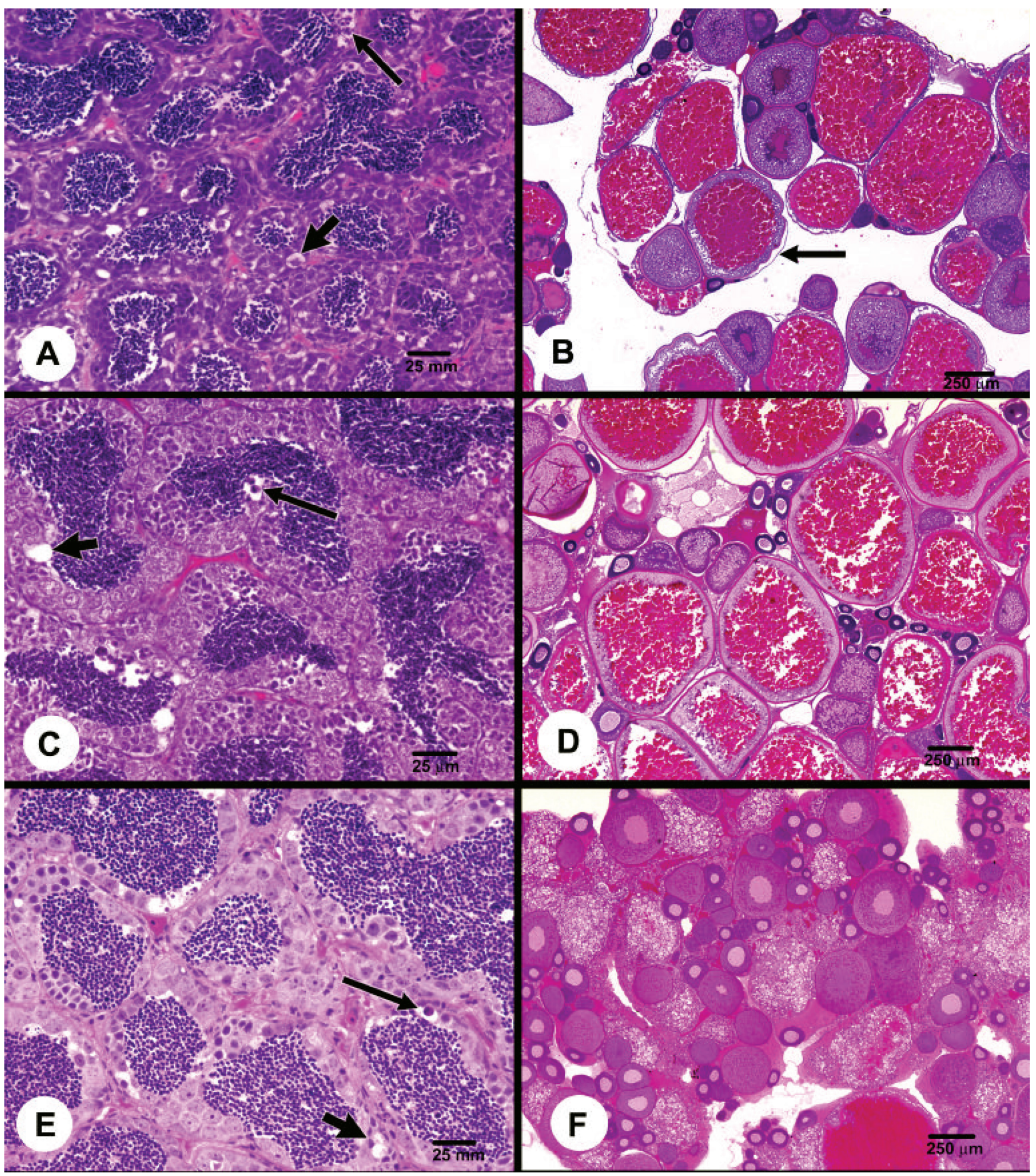

FIGURE 1.-(A) Testis, formalin/paraffin method, H\&E stain. Identification of cell types is seriously hampered by artifactual shrinkage and condensation of nuclei Formalin was considered to be an unsatisfactory fixative for testis cell identification. Note the presence of vacuolated cells (VC $=$ short arrow) and apoptotic body cells $(\mathrm{ABC}=$ long arrow). (B) Ovary, formalin/paraffin method, H\&E stain. This method is adequate for germ cell identification despite minor artifactual changes including dissociation of the follicles, contraction of the oocytes, and wrinkling of the vitelline membrane (arrow). Recognition of early atretic changes is difficult. (C) Testis, Bouin's paraffin method, H\&E stain. Nuclear detail of germinal cells is much improved compared to the formalin/paraffin method, but there is overlap of cells due to the thickness of the tissue section (3-5 $\mu \mathrm{m})$, and cytoplasmic detail is lacking (VC = short arrow; $\mathrm{ABC}=$ long arrow). (D) Ovary, Bouin's/paraffin method, $\mathrm{H} \& \mathrm{E}$ stain. There is excellent contrast and detail, and early atretic changes can be readily identified. For the ovary, this was considered to be the most satisfactory of the evaluated preparation methods. (E) Testis, glutaraldehyde/GMA method, H\&E stain. The thin sectioning (1-2 $\mu$ m) afforded by this method provides superior nuclear and cytoplasmic detail. This level of detail is requisite for the classification of individual spermatogenic cells in situations where the cellular morphology may be altered by exposure of the fish to test compounds $(\mathrm{VC}=$ short arrow; $\mathrm{ABC}=$ long arrow). (F) Ovary, glutaraldehyde/GMA method, $\mathrm{H} \& \mathrm{E}$ stain. Compared to Bouin's/paraffin method, there is diminished color contrast among the various follicle types. Because of the relatively large size of even the smallest follicles, thin sectioning offers little advantage for ovarian cell type identification. 

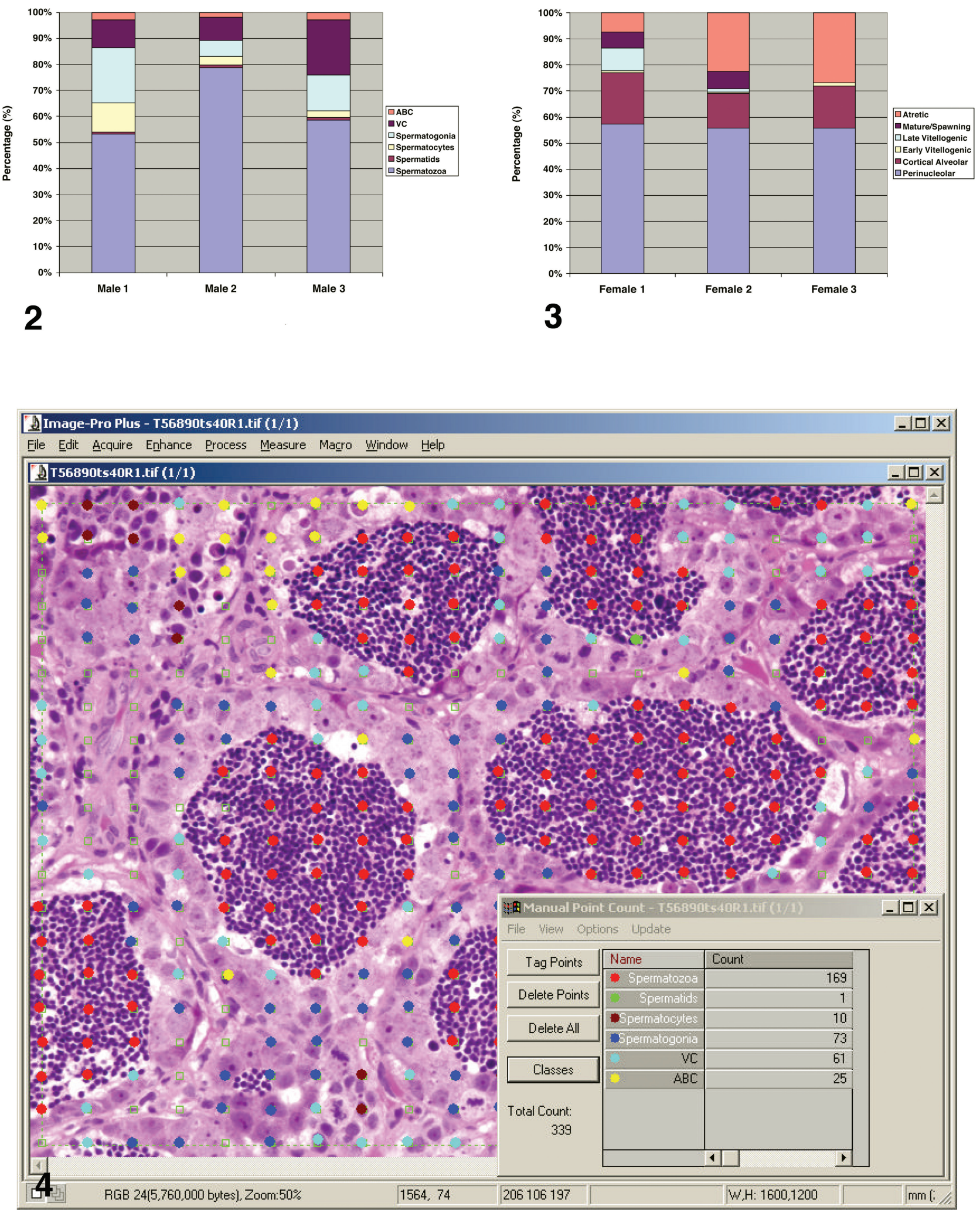

Figures 2-4

FIGURE 2.-Relative percentages of germinal cells in the testes of male fathead minnows exposed to $17 \beta$-estradiol as obtained by manual tagging. 3.-Relative percentages of germinal cells in the ovaries of female fathead minnows exposed to $17 \beta$-estradiol as obtained by manual tagging. 4.- Testis, glutaraldehyde/GMA method, H\&E stain. Manual tagging of digitized photomicrographic images is illustrated. A grid of green boxes extends across the image, and cell-type differentiation is indicated by colored dots that are individually applied to each box using the computer mouse. Boxes that remain untagged represent interstitial areas, areas devoid of tissue, or cells that cannot be reasonably classified due to suboptimal orientation or marked degeneration. Cell counts ( $>2,500$ cells were counted per male fish) are automatically exported to a spreadsheet for tabulation (not shown). 
cytoplasm. Spermatids were present in small clusters or individually within the superficial germinal epithelium; occasionally, spermatids were free within the lobular lumen. Spermatocytes (primary and secondary, approximately 4-8 $\mu \mathrm{m}$ diameters) were usually recognized as well-defined nests of cells within the superficial germinal epithelium. Compared to spermatids, spermatocytes had larger, somewhat less densely basophilic nuclei with a smooth chromatin pattern, inapparent or very faint nucleoli, and small amounts of very pale cytoplasm with polygonal shaped borders. Spermatogonia (approximately $5-10 \mu \mathrm{m}$ in diameter) were present in small clusters or individually within the superficial or deep germinal epithelium (Figure 5). These cells had large, pale, poorly demarcated, open-faced nuclei, nucleoli that tended to be very prominent, and abundant grainy amphophilic cytoplasm that frequently contained scattered small red perinuclear granules (this degree of cellular detail was only apparent in specimens that were preserved in glutaraldehyde and embedded in GMA).

Vacuolated cells $(V C)$ were either observed within the germinal epithelium in random locations or in the lobular lumen as protrusions from the germinal epithelium. VCs were generally large cells that had variable amounts of cytoplasmic vacuolization. At 1 end of the spectrum, some vacuolated cells had 1 to several large clear cytoplasmic vacuoles that did not displace the nucleus (which usually resembled a spermatogonium or spermatocyte nucleus; Figure 5); whereas, at the opposite end of the spectrum, other vacuolated cells had greatly expanded, pale, foamy cytoplasm and marginated, condensed, sharply angular, hyperchromatic nuclei (Figure 6). Apoptotic body cells (ABC) (Figure 5) contained one or more variably sized spherical bodies (basophilic or basophilic with an eosinophilic rim) within one or more large cytoplasmic vacuoles.

As previously mentioned, manual tagging of images acquired from the ovary (Figure 7) utilized Bouin's/paraffin sections. In such sections, perinucleolar follicles (approximately 50-300 $\mu \mathrm{m}$ diameter) were generally the smallest follicles and were characterized by a thick zone of granular, dark basophilic ooplasm that surrounded a sharply defined core of flocculent pale lavender karyoplasm. Often apparent within the margins of the karyoplasm were minute, spherical pink bodies (provitelline nucleoli). Some of the larger perinucleolar follicles had slightly paler ooplasm that contained a scattering of small (less than $10 \mu \mathrm{m}$ diameter) predominately clear, cytoplasmic vacuoles (developing cortical alveoli). Cortical alveolar follicles (approximately 100-500 $\mu \mathrm{m}$ diameter) were generally larger than perinucleolar follicles and were characterized by amphophilic ooplasm that contained numerous, predominately pale pink cytoplasmic bodies (cortical alveoli). The cortical alveoli stage was the first stage in which the refractile eosinophilic vitelline envelope was clearly evident. Nuclei (germinal vesicles) were usually eosinophilic at this stage of development and were often crowded centrally by the cortical alveoli. Early vitellogenic follicles (approximately 250-500 $\mu \mathrm{m}$ diameters) resembled cortical alveolar follicles in size and substance, except that the ooplasm of the former contained a small-to-moderate amount of bright eosinophilic, granular-to-globular material (yolk granules). In late vitellogenic follicles (approximately 500-750 $\mu \mathrm{m}$ diameter), the eosinophilic yolk granules were larger and more numerous compared to those of early vitellogenic follicles, and yolk granules filled the central area of the follicle. The central yolk area of midvitellogenic follicles was enveloped by a zone of blue-gray ooplasm that was greater than $50 \mu \mathrm{m}$ in width. Due to an increase in yolk material, the width of this blue-gray zone was decreased or almost inapparent in mature/spawning follicles (approximately 750 $1,000 \mu \mathrm{m}$ diameter). The most subtle (earliest) indications that follicles were atretic (degenerative) were alterations in the morphologic appearance of the vitelline envelope that became thickened, clumped, less refractile, and developed multiple perforations (Figure 8). These changes were often accompanied by alterations in the adjacent perifollicular cells that included columnar swelling, cytoplasmic vacuolization, and the presence of intracytoplasmic droplets of eosinophilic (yolk) material. Most of the atretic follicles appeared to be in the later stages of vitellogenesis prior to their degeneration, although occasional cortical alveolar follicles and rare perinucleolar follicles were also atretic.

\section{DISCUSSION}

In this study, the FHM were exposed to a high concentration of $17 \beta$-estradiol to simulate a "worst-case scenario" with regard to alterations in gonadal morphology. The dose of the test article ( $10 \mathrm{nM} 17 \beta$-estradiol) and duration of exposure (14 days) were derived from previously published work (Miles-Richardson, 1999a) in which this regimen was shown to produce moderate pathologic alterations in the gonads including degenerative changes and Sertoli cell proliferation (testis) and oocyte atresia (ovary). Notably, the use of this regimen ensured that severe tissue compromise (e.g., overt necrosis) would be avoided, as severe changes might excessively hinder gonad cell identification and possibly affect survival of the fish.

Fathead minnows were chosen as test subjects because they are an archetypal member of the ecologically important and ubiquitous Cyprinidae family, and because they have been used extensively in various short-term assays, chronic lifecycle studies, and early life-stage survival and development tests involving endocrine-active substances in support of regulatory programs in both North America and Europe (Ankley et al., 2001; Jensen et al., 2001; Panter et al., 2002, U.S. EPA, 2002). Reproductive maturity in FHM occurs within 4 to 5 months of hatch under optimal conditions (U.S. EPA, 2002). FHM are fractional spawners; consequently, gametogenesis in both sexes occurs asynchronously, which signifies that the various commonly recognized gametogenic cell types are usually evident simultaneously (U.S. EPA, 2002).

As in most teleosts, the structural organization of the FHM testis is of the unrestricted type, in which spermatogenesis occurs within the germinal epithelium along the entire length of the seminiferous lobule (Grier, 1976; Grier, 1981; Pudney, 1993; U.S. EPA, 2002). The functional unit of the testicular lobule is the spermatocyst, wherein a clonal aggregate of precursor cells in a synchronous phase of development is completely surrounded by the cytoplasmic arms of a single Sertoli cell (Grier, 1993; Pudney, 1993; Patiño and Sullivan, 2002). Following establishment of an association between a spermatogonium and a Sertoli cell, spermatogenesis progresses through a series of mitotic and meiotic divisions that sequentially produce smaller cells and 

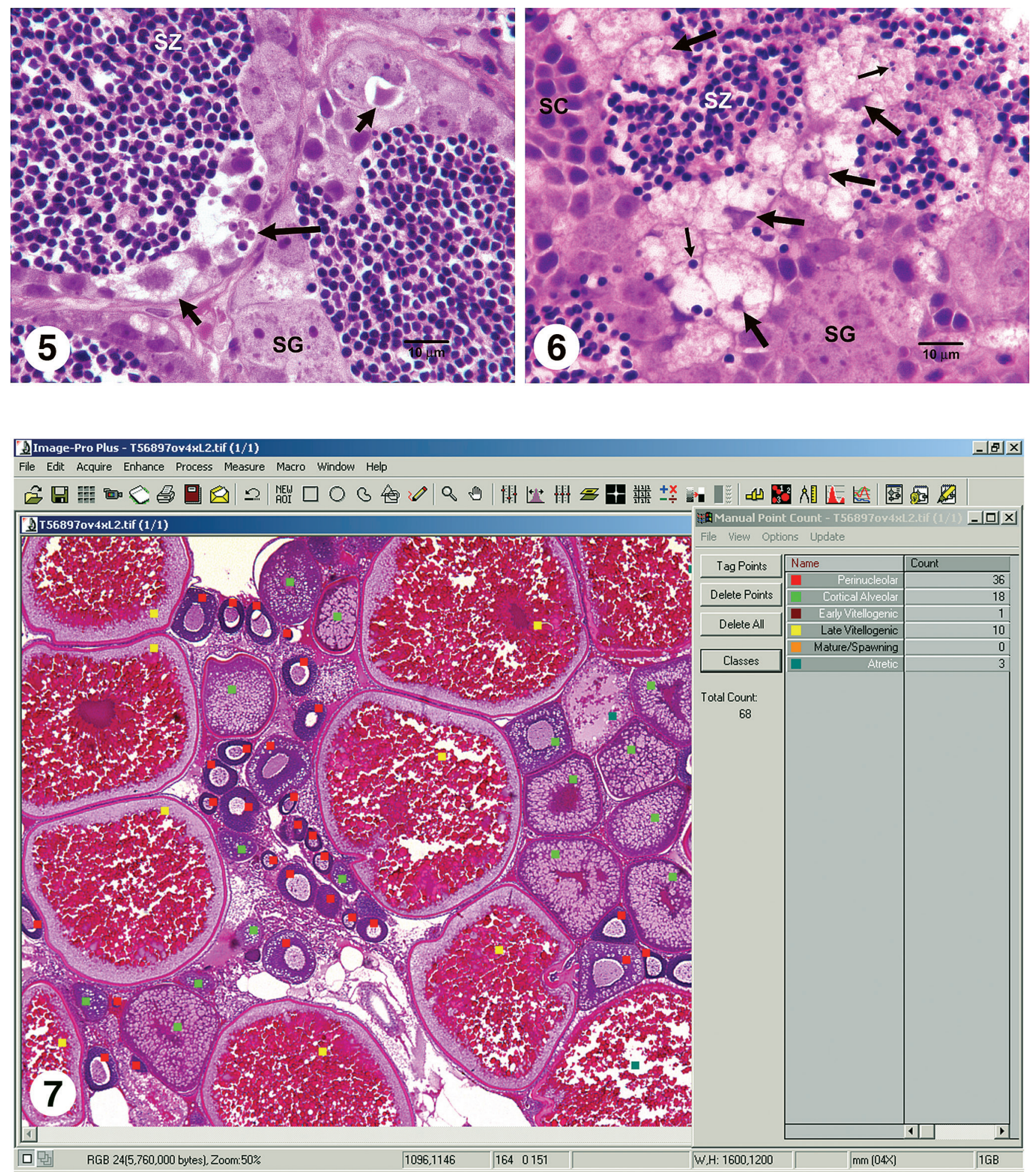

Figures 5-7

FIGURE 5.-Testis, glutaraldehyde/GMA method, H\&E stain. Spermatogonia (SG) have large ovoid nuclei, peripherally clumped chromatin, prominent nucleoli, and moderate-to-abundant amounts of granular cytoplasm. It is presumed that most of these cells are primary and secondary spermatogonia, but some may be hypertrophic Sertoli cells. The long arrow indicates an apoptotic body cell that contains five spherical structures within a large cytoplasmic vacuole. Two other vacuolated cells (VC) in this image (short arrows) are not overtly phagocytic, and some of these cells may be degenerating spermatogonia or spermatocytes. Note that even spermatozoa (SZ) are viewed as a monolayer in 1-2 $\mu \mathrm{m}$ sections. 6.-Testis, glutaraldehyde/GMA method, H\&E stain. The large arrows indicate vacuolated cells (VC) with voluminous foamy cytoplasm and relatively small, angular, condensed nuclei. Some of these cells appear to contain spermatozoa (small arrows) within their cytoplasm. SZ = spermatozoa, $\mathrm{SC}=$ spermatocytes, $\mathrm{SG}=$ spermatogonia. 7.-Ovary, Bouin's/paraffin method, H\&E stain. Manual tagging of digitized photomicrographic images is illustrated. Due to the small number of cells in the ovary relative to the testis, a grid overlay is not utilized, and all germinal cells are counted. A different colored square represents a single stage of follicular development. 


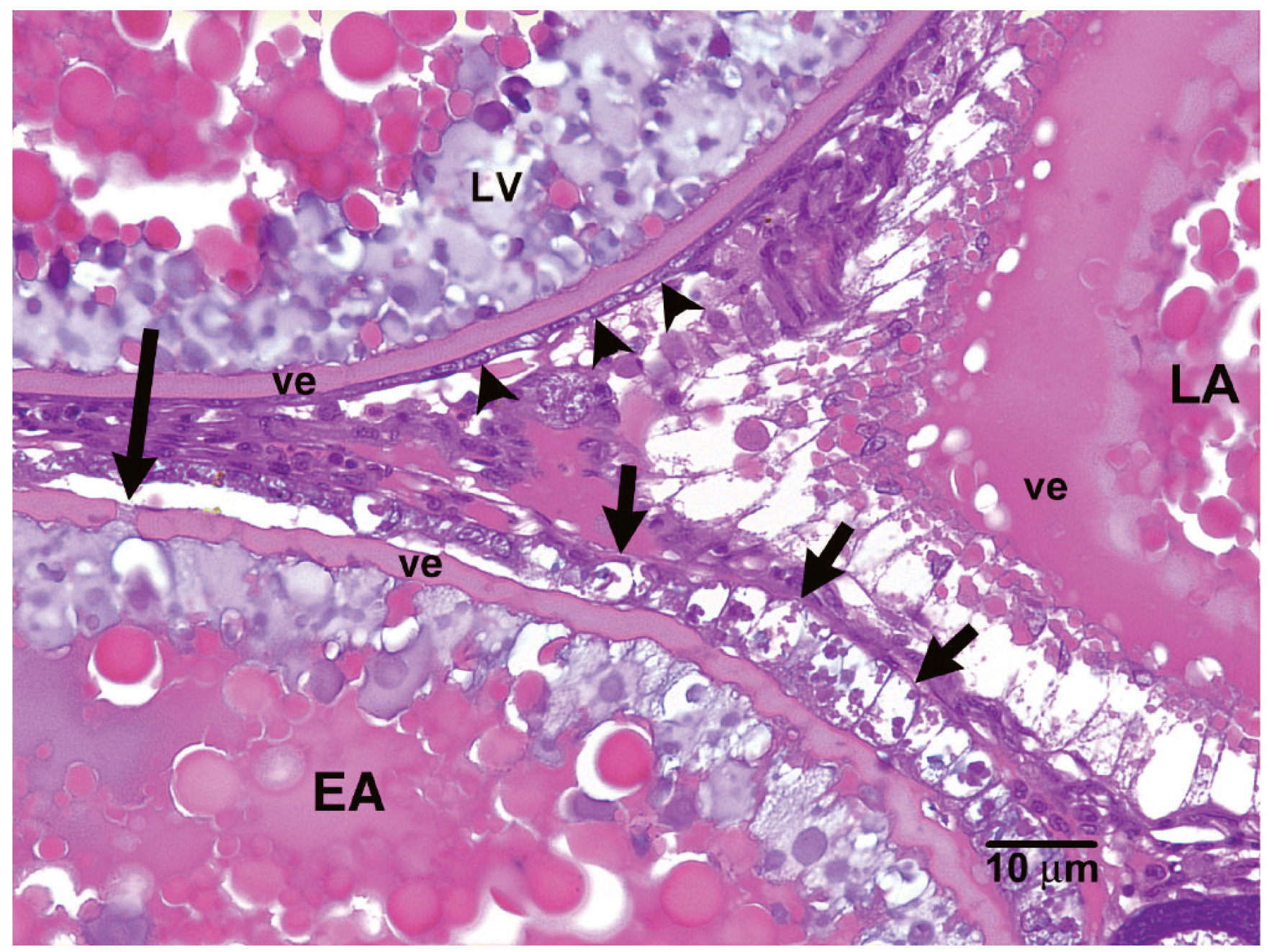

FIGURE 8.-Ovary, Bouin's/paraffin method, H\&E stain. Shown are a late vitellogenic follicle (LV), an early atretic follicle (EA), and a late atretic follicle (LA) follicle. In the early atretic follicle, the vitelline envelope (ve) is clumped and irregular, and small pores are evident (long arrow). Such changes are exaggerated in the late atretic follicle. Note that the perifollicular cells (arrowheads) of the mid-vitellogenic follicle are small and flattened; whereas, the perifollicular cells (short arrows) of the early atretic follicle are columnar, vacuolated, and have intracytoplasmic droplets.

a haploid chromosome configuration according to the following sequence: spermatogonia $\rightarrow$ spermatocytes $\rightarrow$ spermatids (Grier, 1981; Nagahama, 1983; Pudney, 1995; U.S. EPA, 2002). Spermatogonia and spermatocytes are often subdivided into primary and secondary developmental stages (Grier, 1981; Nagahama, 1983; Pudney, 1995). Spermatids undergo maturation events (spermiogenesis) that include nuclear shape changes, development of a flagellum, loss of cytoplasmic volume, and phagocytosis of cytoplasmic remnants (residual bodies) prior to their release into the lobular lumen as spermatozoa (spermiation) (Pudney, 1995; Patiño and Redding, 2000).

In the piscine ovary, the functional unit is the follicle that consists of an oocyte surrounded by perifollicular cells (granulosa cells and cells of the thecal layer) (Nagahama, 1983; Tyler and Sumpter, 1996; Patiño and Redding, 2000). Mitotic and meiotic divisions during oogenesis halve the chromosome number and generate progressively larger cells in the following manner: oogonia $\rightarrow$ perinucleolar (primary growth) oocytes $\rightarrow$ cortical alveolar oocytes $\rightarrow$ early vitel- logenic oocytes $\rightarrow$ late vitellogenic oocytes $\rightarrow$ mature/ spawning oocytes (U.S. EPA, 2002). Some authors have further subdivided early primary growth follicles into additional developmental stages (e.g., bouquet, chromatin-nucleolus, etc.) (Patiño and Redding, 2000). Vitellogenesis, the process by which extra-ovarian proteins produced by the liver are packaged into oocytes, is a hormonally controlled activity that results in tremendous follicular enlargement (Tyler and Sumpter, 1996; Patiño and Sullivan, 2002). Following the maturation period, ovulation occurs when oocytes are shed from within their perifollicular cell sheath and are expelled into the ovarian lumen in arrested phase of the second meiotic division (Patiño and Sullivan, 2002). Follicular atresia, whether caused by physiologic stimuli or environmental stress, entails the degeneration of follicles at any stage of follicular development (Nagahama, 1983) although atretic follicles are often most noticeable in the later developmental stages (Tyler and Sumpter, 1996). Follicular atresia is accompanied by hypertrophy of the perifollicular granulosa cells (Nagahama, 1983). 


\section{Gonad Excision}

The microdissection approach used to excise the FHM male and female gonads was a rapid and straightforward technique that was easily standardized. Despite the diminished size and/or altered texture of some of the gonads from these estradiol-treated FHM, this method consistently yielded intact, well-preserved reproductive organs that were essentially devoid of collection-induced microscopic artifacts. Prior to this experiment, longitudinal (sagittal and parasagittal) or transverse whole fish sectioning was briefly considered as an alternative technique to gonad excision. Although whole fish sectioning might be appropriate for gonad cell quantification in smaller laboratory fishes such as Japanese medaka Oryzias latipes and zebrafish Danio rerio, there are several reasons why such methods are less ideal for larger fishes such as FHM. First, even if the head and the tail were to be removed, standard plastic tissue cassettes are not spacious enough to accommodate the trunk segments of some large adult FHM males if positioned longitudinally.

Although oversize cassettes are available, microtoming the larger paraffin blocks is technically more difficult compared to standard block sizes. Second, because FHM gonads are elongated tubular structures that are oriented essentially parallel to the longitudinal axis of the fish, and since perfectly level embedding of the whole body is difficult to achieve, the entire length of a gonad cannot be consistently recovered on a single slide using longitudinal whole body sectioning. Consequently, a great deal of animal-to-animal variability in both the amount of tissue recovered and the plane of sectioning are to be expected.

Another drawback is that it may be difficult to accurately identify individual gonads as right or left when parasagittal (vertical) whole body sectioning is performed (i.e., if the plane of microtoming is slightly oblique, segments of both gonads may appear in the same tissue section), and at least 2 whole body sections must be prepared per fish if both the right and left gonads are to be evaluated. This drawback would be alleviated if whole fish were sectioned in the frontal (horizontal) plane; however, because FHM normally have an anatomic profile that is laterally compressed, and the dorsal and ventral surfaces of these fish are not parallel to their longitudinal axis, achieving a consistent plane of section would be extremely challenging (if not impossible) using this technique.

On the other hand, if whole fish were microtomed transversely instead of longitudinally, numerous sections would need to be produced and evaluated in order to ensure adequate spatial representation of the gonads. A third reason to prefer gonad excision is that this technique avoids microtoming artifacts that can be caused by the presence of firmer tissues (such as bone and cartilage) that are inherently present in whole body sections. Finally, gonad excision allows these organs to be visualized macroscopically in situ, and it is possible to obtain gonad weights if desired. Concerning the cost-effectiveness of this technique, it should be noted that amount of time required to excise the gonads is only slightly greater than the time that would take to carefully incise the abdomen and flush it with fixative; in addition, decalcification procedures can be omitted if the gonads are excised.

\section{Fixation and Embedding Procedures}

For quantitative gonad staging, the importance of appropriate microdissection procedures and an optimal combination of histological procedures cannot be overemphasized. Compared to semiquantitative grading, in which the relative density of cell populations is subjectively estimated, a higher degree of precision is required to be able to identify and quantify gametogenic precursors on a cell-by-cell basis. This is a prerequisite for the testis, as cellular detail is intrinsically limited by the diminutive size of the germinal cells (the largest spermatogenic cells are approximately $10 \mu \mathrm{m}$ diameter). For the FHM testes in this study, the thin sectioning ( $2 \mu \mathrm{m}$ or less) that is possible with glutaraldehyde-fixed/GMA-embedded sections greatly improved visualization of nuclear and cytoplasmic details in what was essentially a monolayer of cells. Other reported benefits of glutaraldehyde fixation and GMA embedding for preparing FHM gonad sections include improved tissue fixation, reduced shrinkage of sections, reduced distortion due to microtoming, and ease of staining (U.S. EPA, 2002). Because ovarian follicles are vastly larger than spermatogenic cells, thin sectioning was not particularly advantageous for the ovary. Nevertheless, the visualization of early atretic changes in the follicles was enhanced by Bouin's fixation when compared to formalin fixation, which again illustrates that the correct choice of histological procedures is crucial for the accurate assessment of microscopic changes.

The various types of macroscopic and histopathologic findings observed in the male and female FHM gonads in this study (Tables 2 and 3 ) were not considered to be unique to $17 \beta$-estradiol-treated fish; however, it was likely that overall severity and/or prevalence of some of these findings was a consequence of $17 \beta$-estradiol administration, as suggested earlier (Miles-Richardson et al., 1999a). For example, while follicular atresia is considered to be an integral feature of the normal teleost ovarian cycle (Patiño and Redding, 2000) the proportion of atretic follicles identified in these FHM (mean $=18.9 \%$, range $=7.4-26.9 \%, \mathrm{n}=3$ ) was higher than anticipated. Since reported mean percentages of atretic follicles in groups of control FHM have varied from 1.6\% (McCormick et al., 1989) to 5\% (Miles-Richardson et al., 1999b), and it has been speculated that follicular atresia up to 10-12\% may be normal for some FHM (U.S. EPA, 2002). Similarly, vacuolated cells (VC) and apoptotic body cells (ABC) are also frequently present in the testes of FHM that have not been treated with estrogenic compounds (personal observation), although the numbers of such cells in the current study were generally higher than what we would expect to see in untreated fish.

Based on prior experience, the presence of occasional granulomas and mineralized foci in the testes of the male FHM was not surprising; whereas, the minimal to mild sperm necrosis (observed unilaterally or bilaterally in all 3 fish) was considered to be a more unusual finding. Sperm necrosis was also reported to occur in male FHM that were exposed to 4nonylphenol and nonylphenol ethoxylate (Miles-Richardson et al., 1999b). The testis attenuation that was observed at necropsy in some of the male FHM could not be correlated with specific histopathologic lesions; most likely, the attenuation was due to an overall decrease in the cellularity (cell density) of the testes in these particular fish. 
One gonadal alteration that has been reported in other studies is the presence (or relatively increased incidence) of oocytes within the testes of male teleosts that have been exposed to estrogenic substances (Gray and Metcalfe, 1997; Metcalfe et al., 2001). In 1 study, in which a lake was experimentally treated with the synthetic estrogen, ethynylestradiol, the incidence of ovotestis formation in resident male FHM was $11 \%$. (Palace et al., 2002). Oocytes were not observed in the testes of male FHM in the present study, possibly due to the short duration of the exposure (10 days), the exposure of sexually mature fish, and/or the limited number of fish that were examined. For future studies, the manual tagging procedure could be easily adapted to count oocytes that might be present within testis sections.

\section{Overview of FHM Testis and Ovary Cell Types}

Prior to manually tagging the gonadal cells in digital images, it was necessary to define the morphologic criteria for cell categorization. Although various descriptions of FHM gametogenic cells have been published, (Miles-Richardson et al., 1999a), none were considered to be completely satisfactory for counting individual cells at the light microscopic level. Regarding the testis, cell categorization was more challenging than anticipated, as there appeared to be disparity in the scientific literature concerning classification criteria. Particularly problematic was the issue of Sertoli cell histomorphology. While there is considerable information pertaining to Sertoli cell-germ cell interactions in mammalian testis (Russell and Griswold, 1993), and while the fundamental roles of mammalian and fish Sertoli cells appear to be comparable (Russell and Griswold, 1993), only limited data were available for fish in general (Grier, 1993); and fish demonstrated far more functional and morphological diversity in their reproductive strategies when compared to mammals (Grier, 1993). Of special importance in the present context was the fact that abnormally large Sertoli cells (hypertrophy), often in parallel with increased numbers (hyperplasia), have been associated with exposure to some estrogenically active compounds (Miles-Richardson et al., 1999a, 1999b; Kinnberg et al., 2000; van der Ven et al., 2002; Kinnberg and Toft, 2003).

Although there is a consensus description among various sources in which the normal Sertoli cells of various osteichthyes are characterized by small pyramidal-shaped or elongated nuclei and minimal or attenuated cytoplasm (Pudney, 1993; Miles-Richardson et al., 1999a; Kinnberg et al., 2000; U.S. EPA, 2002), the light microscopic appearance of altered Sertoli cells as represented in the scientific literature is ambiguous. For example, in 1 report in which Sertoli cell hypertrophy in FHM was considered to be a consequence of $17 \beta$-estradiol exposure, the hypertrophic Sertoli cells were depicted as having a "vesiculate nucleus and a prominent nucleolus" (Miles-Richardson et al., 1999a). Based on this description and associated photographs, the enlarged Sertoli cells in this paper had a polygonal shape and appeared virtually indistinguishable from spermatogonia. Pudney, for example, characterized spermatogonia as having "large regular nuclei usually containing a distinct nucleolus" (Pudney, 1995).

Conversely, in the testes of zebrafish Danio rerio exposed to $17 \alpha$-methyldehydrotestosterone (van der Ven et al., 2003), the hypertrophic Sertoli cells appeared to be proportionally smaller cells that had ovoid to reniform nuclei, minimal obvious cytoplasm, and variably evident nucleoli. In another study, "hypertrophic cuboidal to columnar Sertoli cells with spherical nuclei" were observed in the testes of control platyfish Xiphophorus maculatus, and increased numbers of these cells were present in the testes of platyfish that had been exposed to nonylphenol (Kinnberg et al., 2000). Concerning the physiologic hypertrophy of Sertoli cells as is reported to occur in poecilids, these cells have also been described as columnar (Pudney, 1995). In the photomicrographs that accompanied these reports, the hypertrophic Sertoli cells of zebrafish and platyfish bore little resemblance to spermatogonia.

A measure of inconsistency in the characterization of hypertrophic Sertoli cells is undoubtedly due to interspecies differences. It is also possible that the hypertrophic appearance of some Sertoli cells may be dependent on the nature of the cytomegalic stimulus (i.e., the morphology of these cells may vary according to the physiological requirements of the testis during a given period of development or according to the type of exogenous chemical exposure). An additional consideration is the fact that piscine Sertoli cells have few defining light microscopic features. Even at the ultrastructural level, justification for the homologous existence of Sertoli cells in fishes is primarily dependent upon the finding of residual spermatogenic materials in the cytoplasm of such cells and the presence of junctional complexes that define the Sertoli cell barrier (Grier, 1981, 1993). Thus, it is not surprising that fish Sertoli cells have been confused with other cell types, such as spermatogonia, when viewed by light microscopy (Pudney, 1993).

Currently, ultrastructural analysis appears to be the method of choice for the definitive identification of piscine Sertoli cells. Unfortunately, electron microscopy is an impractical tool for assessing cell proportions because the number of cells that can be viewed at any one time is very limited. Until further standards of recognition become available (e.g., the identification of Sertoli cells via routine immunohistochemical staining), the most rational approach may be to utilize descriptive terminology for those cells that cannot be associated with well-defined cell categories by light microscopy. On this basis, and for the purpose of cell counting in this study, the label "spermatogonium" was applied to cells that possessed particular morphologic features, including large overall size, large, poorly demarcated, open-faced nuclei, prominent nucleoli, eosinophilic perinuclear granules, and moderate-tolarge amounts of grainy cytoplasm (Figures 4 and 5). It is likely that the vast majority of these cells were spermatogonia (primary and secondary); however, based on various descriptions and photographs in the scientific literature, it is also possible that at least some of these were hypertrophic Sertoli cells. For each of the 3 separate $17 \beta$-estradiol-treated male fish in this study, a potentially important result from the manual tagging exercise was the relative predominance of spermatogonia when compared to spermatocytes and spermatids (Figure 2). It should also be noted that relatively low numbers of small, angular cells consistent with nonhypertrophic Sertoli cells were recognized during the manual tagging procedure but were not specifically tagged.

In addition to ambiguity regarding the morphology of hypertrophic Sertoli cells, the categorization of testis cell types 
was challenging because previously established staging systems did not specifically address other forms of histomorphologically altered cells. In particular, 2 types of altered cells that were observed in this study were termed vacuolated cells (VC) and apoptotic body cells (ABC). The cellular origins of $\mathrm{VC}$ and $\mathrm{ABC}$ were not always obvious during light microscopic examination; consequently, to justly labeling such cells, descriptive appellations such as VC and ABC were created for cell counting purposes. The general appearance of many VC (those with 1 to several large, clear, cytoplasmic vacuoles that did not displace the nucleus) strongly suggested that they were vacuolated spermatogonia or spermatocytes that had undergone degenerative changes as a consequence of $17 \beta$-estradiol exposure (Figure 4). It is quite possible that other VC (those with greatly expanded, pale, foamy cytoplasm) were derived from a different cell type (e.g., activated macrophages or Sertoli cells) (Figure 5).

The presence of large vacuoles in Sertoli cells has been attributed to phagocytosis of residual bodies and other cellular elements (Loir et al., 1995). Although it could be contended that $\mathrm{VC}$ were artifacts of tissue handling or processing in this experiment, this notion is difficult to defend as VC (and $\mathrm{ABC}$ ) were evident in all 3 fixation/embedding combinations and in each testis to varying degrees (Figure 1). The existence of apoptotic bodies within the cytoplasmic vacuoles of some cells (ABC) supports the concept that phagocytic cells are present (and probably resident) within the testicular lobule. It is relevant that among Chordata, a unifying function of Sertoli cells, is the phagocytosis of effete spermatozoa and spermatogenesis by-products (Grier, 1993; Russell and Griswold, 1993; Loir et al., 1995), and that degenerate and presumptively apoptotic sperm have been observed ultrastructurally within the Sertoli cells of FHM and other fishes (Grier, 1993; Miles-Richardson et al., 1999a, 1999b).

\section{Manual Tagging of Individual Cell Types}

Automated image analysis techniques, in which the identification of individual cell types is performed by computer software using colorimetric and morphometric thresholds, were given initial consideration for this study. It is reasonable to assume that the throughput advantage of automated counting would be significant as compared to manual counting, and that it might be possible to enhance cell type discrimination for automated counting through the use of immunohistochemical procedures. Ultimately, automated analysis techniques were not employed, however, because certain gonadal cell types (spermatogonia, $\mathrm{VC}, \mathrm{ABC}$, and many atretic follicles) would have been difficult for the image analysis software to recognize as distinct entities, due to the variability and subtlety of the structural changes in these cells.

A different manual approach to quantitative testis cell evaluation was recently reported by van der Ven, Wester, and Vos (2003). In their study, which utilized zebrafish Danio rerio, clonal aggregates of spermatogenic cells (spermatocysts) were outlined in digitized images, and the 2-dimensional areas of the outlined regions were then measured. Although this appeared to be an elegant approach for the zebrafish testes, it would have been difficult to apply this particular technique in the present study, because the organizational pattern of the FHM spermatocysts appeared to be disrupted (i.e., spermatocysts were generally small and very poorly defined), presumably as a result of $17 \beta$-estradiol treatment. Additionally, cell types that were present individually or in small aggregates in the FHM testes (such as ABC and VC) would not have been recognized and counted using the van der Ven, Wester, and Vos method. On the other hand, due to microanatomical interspecies differences, the manual tagging protocol employed in the present study could not be readily utilized for certain other fishes without modification. An example would be atheriniform fishes such as the Japanese medaka Oryzias latipes. Unlike FHM, in which the cell type distribution tends to be relatively homogenous throughout the length and width of the testis, spermatogenic primordia are restricted to the periphery of the testis in medaka (Grier, 1976). Because the distribution of spermatogenic cells is nonuniform in medaka, it is unlikely that a single longitudinal tissue section would adequately represent the cellular composition of an entire medaka testis.

As performed in the present study, the manual tagging of individual cell types in digital images afforded a rigorous technique for the evaluation of potential changes in gonad cell proportions, as have been reported to occur following exposure of fish to certain xenobiotic chemicals. Contrasted to a previous study in which FHM testis cells were counted during direct microscopic visualization of tissue sections (Sohoni et al., 2001), the image-based manual tagging technique provided a permanent and reviewable record of each cell counted. Additionally, the manual tagging technique provided benefits that may not be obvious at first glance. For example, the grid markings that were applied to each glass slide, and the virtual grid that was applied to each image, effectively prevented duplicate evaluation of testis areas and duplicate cell counting, respectively. The ability to magnify each image to the limits of image resolution was conducive to the detailed morphological inspection of the gonadal cell types. Representative sampling of the gonads was achieved by counting large numbers of cells for each fish $(>2,500$ cells per male and $>250$ cells per female).

The classification systems that were employed to identify and quantify gonadal cells utilized a flexible combination of established and descriptive terminology; thus, cells that may have been altered by $17 \beta$-estradiol exposure and therefore were difficult to associate with defined cell categories of testicular development were readily incorporated as descriptive cell categories (VC, ABC). Finally, the light microscopic evaluation of individual gametogenic cells was greatly facilitated by specimen preparation techniques that included the excision of gonads via microdissection and by optimized fixation and embedding procedures (5\% glutaraldehyde/GMA for the testis and Bouin's solution/paraffin for the ovary). Results of this pilot study indicate that quantitative staging by manual tagging is a feasible and meticulous scientific tool for the comparative histological assessment of fathead minnow gonads.

\section{REFERENCES}

Ankley, G. T., Jensen, K. M., Kahl, M. D., Korte, J. J., and Makynen, E. A. (2001). Description and evaluation of a short-term reproduction test with the fathead minnow (Pimephales promelas). Environ Toxicol and Chemi 20(6), 1276-90. 
Ankley, G. T., Kahl, M. D., Jensen, K. M., Hornung, M. W., Korte, J. J., Makynen, E. A., and Leino, R. L. (2002). Evaluation of the aromatase inhibitor fadrozole in a short-term reproduction assay with the fathead minnow (Pimephales promelas). Toxicol Sci 67, 121-30.

Christiansen, T., Korsgaard, B., and Jespersen, $\AA$. (1998). Effects of nonylphenol and $17 \beta$-estradiol on vitellogenin synthesis, testicular structure and cytology in male eelpout (Zoarces viviparus). J Exp Biol 201, 17992.

Department of the Interior, U.S. Geological Survey, Biological Resources Division. (2000). U.S. Biomonitoring of Environmental Status and Trends (BEST) Program: Selected Methods for Monitoring Chemical Contaminants and Their Effects in Aquatic Ecosystems. Information and Technology Report, pp. 30-42 USGS/BRD/ITR-2999-0005. Washington, DC.

Gray, M. A., and Metcalfe, C. D. (1997). Induction of testis-ova in Japanese medaka (Oryzias latipes) Exposed to $p$-Nonylphenol. Environ Toxicol and Chem 16, 1082-6.

Grier, H. J. (1976). Sperm development in the teleost Oryzias latipes. Cell Tiss Res 168, 419-31.

Grier, H. J. (1981). Cellular organization of the testis and spermatogenesis in fishes. Am Zool 21, 345-57.

Grier, H. J. (1993). Comparative organization of Sertoli cells including the Sertoli cell barrier. In The Sertoli Cell (L. D. Russell and M. D. Griswold, eds.), pp. 704-739. Cache River Press, Clearwater, FL.

Jensen, K. M., Korte, J. J., Kahl, M. D., Pasha, M. S., and Ankley, G. T. (2001). Aspects of basic reproductive biology and endocrinology in the fathead minnow (Pimephales promelas). Comp Biochem Physiol C Toxicol Pharmacol. 128, 127-41.

Kinnberg, K., Korsgaard, B., Bjerregaard, P., and Jespersen, Å. (2000). Effects of nonylphenol and $17 \beta$-estradiol on vitellogenin synthesis and testis morphology in male platyfish (Xiphophorus maculatus). J Exp Biol 203, 17181.

Kinnberg, K., and Toft, G. (2003). Effects of estrogenic and antiandrogenic compounds on the testis structure of the adult guppy (Poecilia reticulata). Ecotoxicol Environ Saf 54, 16-24.

Loir, M., Sourdaine, P., Mendis-Handagama, S. M. L. C., and Inra, B. J. (1995). Cell-cell interactions in the testis of teleosts and elasmobranchs. Microsc Res Tech 5(32), 533-52.

McCormick, J. M., Stokes, G. N., and Hermanutz, R. O. (1989). Oocyte atresia and reproductive success in fathead minnows (Pimephales promelas) exposed to acidified hardwater environments. Arch Environ Contam Toxicol 18, 207-14.

Metcalfe, C. J., Croley, T. R., March, R. E., and Potter, T. (2001). Estrogenic potency of chemicals detected in sewage treatment plant effluents as determined by in vivo assays with Japanese medaka (Oryzias latipes). Environ Toxicol Chem 20, 297-308.

Miles-Richardson, S. R., Kramer, V. J., Fitzgerald, S. D., Render, J. A., Yamini, B., Barbee, S. J., and Giesy, J. P. (1999a). Effects of waterborne exposure of $17 \beta$-estradiol on secondary sex characteristics and gonads of fathead minnows (Pimephales promelas). Aquat Toxicol 47, 129-45.

Miles-Richardson, S. R., Pierens, S., Nichols, K. M., Kramer, V. J., Snyder, E. M., Snyder, S. A., Render, J. A., Fitzgerald, S. D., and Giesy, J. P. (1999b). Effects of waterborne exposure to 4-nonylphenol and nonylphenol ethoxylate on secondary sex characteristics and gonads of fathead minnows (Pimephales promelas). Environ Res 80, S122S137.
Nagahama, Y. (1983). The functional morphology of teleost gonads. In Fish Physiology (W. S. Hoar, D. J. Randall, and E. M. Donaldson, eds.), pp. 223 275. Academic Press, San Diego, CA

Nichols, K. M., Miles-Richardson, S. R., Snyder, E. M., and Giesy, J. P. (2001). Effects of exposure to municipal wastewater in situ on the reproductive physiology of the fathead minnow (Pimephales promelas). Environ Toxicol and Chem 18, 2001-12.

Palace, V. P., Evans, R. E., Wautier, K., Baron, C., Vandenbyllardt, L., Vandersteen, W., and Kidd, K. (2002). Induction of vitellogenin and histological effects in wild fathead minnows from a lake experimentally treated with the synthetic estrogen, ethynylestradiol. Water Qual Res J Canada 37, $637-50$.

Panter, G. H., Hutchinson, T. H., Länge, R., Lye, C. M., Sumpter, J. P., Zerulla, M., and Tyler, C. R. (2002). Utility of a juvenile fathead minnow screening assay for detecting (anti-) estrogenic substances. Environ Toxicol Chem $\mathbf{2 1}$, $319-26$.

Patiño, R., and Redding, J. M. (2000). Reproductive systems. In The Laboratory Fish (G. K. Ostrander, ed.), pp. 489-500. Academic Press, San Diego, CA.

Patiño, R., and Sullivan, C. V. (2002). Ovarian follicle growth, maturation, and ovulation in teleost fish. Fish Physiol Biochem 26, 57-70.

Pudney J. (1993). Comparative cytology of the non-mammalian vertebrate Sertoli cell. In The Sertoli Cell (L. D. Russell and M. D. Griswold, eds.), pp. 612-657. Cache River Press, Clearwater, FL.

Pudney, J. (1995). Spermatogenesis in nonmammalian vertebrates. Microsc Res Tech 32, 459-97.

Russell, L. D., and Griswold, M. D. (1993). The Sertoli Cell. Cache River Press, Clearwater, FL

Smith, R. J. F. (1978). Seasonal changes in the histology of the gonads and dorsal skin of the fathead minnow, Pimephales promelas. Can J Zool 56, 2103-9.

Sohoni, P., Tyler, C. R., Hurd, K., Caunter, J., Hetheridge, M., Williams, T., Woods, C., Evans, M., Toy, R., Gargas, M., and Sumpter, J. P. (2001). Reproductive effects of long-term exposure to bisphenol A in the fathead minnow (Pimephales promelas). Environ Sci Tech 35, 2917-25.

Tyler, C. R., and Sumpter, J. P. (1996). Oocyte growth and development in teleosts. Rev Fish Biol Fisheries 6, 287-318.

U.S. Environmental Protection Agency. (2002). A Short-term Test Method for Assessing the Reproductive Toxicity of Endocrine-Disrupting Chemicals Using the Fathead Minnow (Pimephales promelas). EPA 600/R-01-067, pp. 141, 144. Washington, DC.

Van den Belt, K. P., Wester, W., van der Ven, L. T. M., Verheyen, R., and Witters, H. (2002). Effects of ethynylestradiol on the reproductive physiology in zebrafish (Danio rerio): time dependency and reversibility. Environ Toxicol and Chem 21, 767-75.

van der Ven, L., and Wester, P. (2002). Normal histology and effects of hormonal active agents of reproductive organs of the zebrafish (Danio rerio). OECD Technical Workshop on Gonadal Histology of Small Laboratory Fishes as a Tool for Endocrine Disruption Screening and Testing. OECD Bilthoven, Netherlands.

van der Ven, L. T. M., Wester, P. W., and Vos, J. G. (2003). Histopathology as a tool for the evaluation of endocrine disruption in Zebrafish (Danio rerio). Environ Toxicol Chem 22, 908-13.

Yonkos, L. T., Kane, A. S., and Reimschuessel, R. (2000). Fathead minnow histology atlas: worldwide web outreach and utilization. Mar Environ Res 50, $1-5$. 